\title{
PRIMITIVE ROOTS IN QUADRATIC FIELDS
}

\author{
JOSEPH COHEN
}

\begin{abstract}
We consider an analogue of Artin's primitive root conjecture for units in real quadratic fields. Given such a nontrivial unit, for a rational prime $p$ which is inert in the field the maximal order of the unit modulo $p$ is $p+1$. An extension of Artin's conjecture is that there are infinitely many such inert primes for which this order is maximal. This is known at present only under the Generalized Riemann Hypothesis. Unconditionally, we show that for any choice of 7 units in different real quadratic fields satisfying a certain simple restriction, there is at least one of the units which satisfies the above version of Artin's conjecture.
\end{abstract}

\section{INTRODUCTION}

A natural question to ask is if there are many primes for which 2 is a primitive root, that is if the subgroup $\langle 2\rangle$ of the multiplicative group $\mathbb{F}_{p}^{\star}$ of the field of $p$ elements generated by 2 is the whole group. Is there a finite number of such groups $\mathbb{F}_{p}^{\star}$ ? Does the same apply for any integer $a$ ?

In 1927 Emil Artin made the following conjecture ([1]):

Conjecture 1.1. Let $a \neq-1$ be an integer which is not a perfect square. Then there are infinitely many primes $p$ such that

$$
<a>=\mathbb{F}_{p}^{\star} .
$$

In addition, for $x>0$, the number of primes $p \leq x$ with this property is asymptotic as $x \rightarrow \infty$ to

$$
A(a) \frac{x}{\log x}
$$

where $A(a)$ is a constant which depends on a.

In 1967 Hooley proved ([8]) Artin's conjecture with the asymptotic formula under the Generalized Riemann Hypothesis. In 1983 Gupta and Murty (4) proved that there are 13 specific integers such that at

Date: July 23, 2018.

Submitted in partial fulfillment of the requirements for the degree of Doctor of Philosophy. Supported by grants from the Technion-Israel Institute of Technology. 
least one of them fulfills the Artin conjecture. From the proof we can deduce that Artin's conjecture is true for almost all integers. R. Murty, K. Murty and Gupta ([5]) showed that we can reduce the specific set of integers from 13 to 7 . Improving the analytic part of Gupta and Murty enabled Heath-Brown to give the best result till now:

Theorem 1.2. Let $q, r$ and $s$ any three primes. Then at least one of them is a primitive root mod $p$ for infinitely many primes $p$.

We note that theorem 1.2 holds for any three non-zero integers, $q, r$ and $s$ which are multiplicatively independent where $q, r, s,-3 q r,-3 q s$, $-3 r s$ and $q r s$ are not a square. (we say that $r$ integers $a_{1}, \ldots, a_{r}$ are multiplicatively independent if for any integers $n_{1}, \ldots, n_{r}, a_{1}^{n_{1}} \cdots a_{r}^{n_{r}}=$ $\left.1 \Rightarrow n_{1}=\ldots=n_{r}=0\right)$.

In this work we present an analog of Artin's conjecture in a different field and we will prove a result similar to the one just shown (we will show that a set which contains a specific number of elements or more always contains a primitive root).

1.1. Artin's conjecture in a real quadratic field. Let $d \neq 1$ be a square-free natural number and let $\Delta=d$ if $d \equiv 1(\bmod 4)$ and $\Delta=4 d$ otherwise. Let $K=\mathbb{Q}(\sqrt{\Delta})$ be a real quadratic field and denote the integer ring of $K$ by $\mathcal{O}_{K}$. The principal ideals that are generated by a rational prime $p, p \mathcal{O}_{K}$ take one of the following forms

(1) $p \mathcal{O}_{K}=P$ (inert);

(2) $p \mathcal{O}_{K}=P_{1} P_{2}, \quad P_{1} \neq P_{2}$ (splits);

(3) $p \mathcal{O}_{K}=P^{2}$ (ramified)

where $P$ and $P_{i}$ are prime ideals in $\mathcal{O}_{K}$. We note that the option (3) occurs only in a finite number of cases and so does not interest us.

Now, the norm map

$$
\mathcal{N}: \mathcal{O}_{K} \mapsto \mathbb{Z}
$$

gives a homomorphism

$$
\mathcal{O}_{K} /(p) \mapsto \mathbb{Z} / p
$$

For any unit $\epsilon$ the kernel of this map contains the residue class $\epsilon$ modulo $p$. Denote this kernel by $C_{\epsilon}(p)$. By lemma 19 in [10] (appendix B)

$$
\operatorname{ord}\left(C_{\epsilon}(p)\right)= \begin{cases}p-1, & p \text { splits } \\ p+1, & p \text { inert }\end{cases}
$$

Assuming GRH, Cooke and Weinberger ([3] ) and Lenstra ([1] ) showed that given a real quadratic field $K$, there are infinitely many split 
primes for which the fundamental unit of the field ${ }^{1}$ has maximal order (namely $p-1$ ) in $C_{\epsilon}(p)$.

Using the strong analytic theorem of Heath-Brown [8], Narkiewicz 14] proved the following unconditional theorem:

Theorem 1.3. Let $\epsilon_{1}, \epsilon_{2}, \epsilon_{3}$ be units in the integers rings $\mathcal{O}_{K_{1}}, \mathcal{O}_{K_{2}}, \mathcal{O}_{K_{3}}$ of $K_{1}=\mathbb{Q}\left(\sqrt{\Delta_{1}}\right), K_{2}=\mathbb{Q}\left(\sqrt{\Delta_{2}}\right), K_{3}=\mathbb{Q}\left(\sqrt{\Delta_{3}}\right)$, respectively, which are not roots of unity. There is an index $j, 1 \leq j \leq 3$, such that for infinitely many split primes $p, c_{j} \epsilon_{j}, c_{j}= \pm 1$, has order $p-1(\bmod (p))$.

For inert primes, one wants similar results. On GRH, an analogue of 3] 11 was only proven recently by Roskam (15). We want to extend the result of Narkiewicz for inert primes.

In this case the order of $C_{\epsilon}(p)(\bmod p)$ is $p+1$. So we cannot use the the result of Heath-Brown on the divisors of $p-1$. We shall use a simpler method to get infinitely many primes $p$ such that $\frac{p+1}{2}=P_{3}$ (we write $P_{3}$ for an integer with at most three prime factors) but with almost same magnitude of the prime divisors. With this result we obtain:

Theorem 1.4. Let $\epsilon_{1}, \ldots, \epsilon_{7}$ be units in the rings of integers $\mathcal{O}_{\Delta_{1}}, \ldots$, $\mathcal{O}_{\Delta_{7}}$ of $\mathbb{Q}\left(\sqrt{\Delta_{1}}\right), \ldots, \mathbb{Q}\left(\sqrt{\Delta_{7}}\right)$, respectively, which are not roots of unity, with $\Delta_{1}, \ldots, \Delta_{7}$ multiplicatively independent and distinct from 3. Assume that all the numbers $(-1)^{a_{1}} 3^{a_{2}} \prod_{i=1}^{7} \Delta_{i}^{b_{i}}, a_{i}, b_{i} \in\{0,1\}$, are not perfect squares if $\sum_{i=1}^{7} b_{i}$ is odd. Then there exists an index $1 \leq j \leq 7$, such that for infinitely many inert primes $p$, the unit $c_{j} \epsilon_{j},\left(c_{j}= \pm 1\right)$, has order $p+1$ modulo $p \mathcal{O}_{\Delta_{j}}$.

Corollary 1.5. Let $\epsilon_{1}, \ldots, \epsilon_{7}$ be units in the rings of integers $\mathcal{O}_{\Delta_{1}}, \ldots$, $\mathcal{O}_{\Delta_{7}}$ of $\mathbb{Q}\left(\sqrt{\Delta_{1}}\right), \ldots, \mathbb{Q}\left(\sqrt{\Delta_{7}}\right)$, respectively, which are not roots of unity, with $\Delta_{1}, \ldots, \Delta_{7}$ primes distinct from 3 . Then there exists an index $1 \leq j \leq 7$, such that for infinitely many inert primes $p$, the unit $c_{j} \epsilon_{j}$, $\left(c_{j}= \pm 1\right)$ has order $p+1$ modulo $p \mathcal{O}_{\Delta_{j}}$.

\section{The work of Gupta-Murty and of Heath-Brown}

Since our work is based on the idea of Gupta and Murty with the advanced version as in the paper of Heath-Brown it will be natural to present their work. We start with following trivial idea: Since the

\footnotetext{
${ }^{1}$ assume that it has norm +1
} 
number of elements in $\mathbb{F}_{p}^{\star}$ is $p-1$, if we show for all integer $d$, and infinitely many primes $p$

$$
d \mid p-1, d \neq p-1 \Rightarrow a^{d} \neq 1 \bmod p
$$

we will have proven the conjecture.

So our first goal is to find infinitely many primes $p$ with a small number of prime divisors of $p-1$. Heath-Brown proved the following lemma.

Lemma 2.1. Let $k=1,2$ or 3 and $K=2^{k}$. Let $q, r$ and $s$ be any three primes. Then for any sufficiently large $x \in \mathbb{R}^{+}$there exist two numbers $\epsilon, \delta \in(0,1 / 4)$ and $c=c(\epsilon, \delta)>0$ so that there are at least $c \frac{x}{\log ^{2} x}$ primes $p \leq x$ which satisfy:

Either $\frac{p-1}{K}$ is prime or $\frac{p-1}{K}=p_{1} p_{2}$ for $p_{1}, p_{2}$ primes $p_{i}>p^{1 / 4+\epsilon}, i=$ 1,2 and $p_{1}<p^{1 / 2-\delta}$. Furthermore, $p$ satisfies

$$
\left(\frac{q}{p}\right)=\left(\frac{r}{p}\right)=\left(\frac{s}{p}\right)=-1 .
$$

Now we prove theorem 1.2 from this lemma. Assume for simplicity that $K=2$ and that we have infinitely many primes $p \leq x$ as in the lemma 2.1] such that $\frac{p-1}{2}=l$ where $l$ is a prime. Take one of the three primes in the lemma, say, $q$. If the order of $q$ equals $l$ we get a contradiction to the fact that $\left(\frac{q}{p}\right)=-1$ by the theorem on cyclic groups (the order of the squares subgroup of $\mathbb{F}_{p}^{\star}$ is $\frac{p-1}{2}=l$ ). If $\operatorname{ord}(q)=2 l$ we are done. If not, the only possibility left is $\operatorname{ord}(q)=2$ but this does not occur for sufficiently large primes $p$ and hence $q$ is a primitive root.

Assume now that there exist $c \frac{x}{\log ^{2} x}$ primes $p \leq x$ as in lemma 2.1 such that $\frac{p-1}{2}=p_{1} p_{2}$. As before the order of $q, r$ and $s$ can be (if they are not primitive) $2,2 p_{1}$ or $2 p_{2}$. As before there is only a small number of cases where $\operatorname{ord}(q)=2$. Assume that $\operatorname{ord}(q)=2 p_{1}$. For this case we need some observation. Let $n$ be a natural number and $\Omega(n)$ denote the number of prime factors of $n$ (with multiplicity) and write $f \ll g$ (or $g \gg f$ or $f=O(g)$ ), where $g$ is a positive function, if there exists a constant $c>0$ such that $|f(x)| \leq c g(x)$. Then

Observation 2.2. (1) For any natural number $n, \Omega(n) \ll \log n$.

(2) Given an integer $a$, The number of primes $p$ such that $\operatorname{ord}(a)<$ $y(\bmod p)$ is $O\left(y^{2}\right)$.

To see (1), use $n=q_{1}^{\alpha_{1}} \cdots q_{r}^{\alpha_{r}} \geq 2^{\alpha_{1}} \cdots 2^{\alpha_{r}} \geq 2^{\alpha_{1}+\ldots+\alpha_{r}}=2^{\Omega(n)}$. To see $(2)$, use $\sum_{m<y} \Omega\left(a^{m}-1\right) \ll_{a} \sum_{m<y} \log \left(a^{m}-1\right) \ll_{a} \sum_{m<y} m \ll_{a} y^{2}$.

Now, if $\operatorname{ord}(q)=2 p_{1}<x^{1 / 2-\delta}(\bmod p)$, then by observation 2.2 this occurs for at most $\left(x^{1 / 2-\delta}\right)^{2}=x^{1-2 \delta}$ primes. 
Since $\frac{x^{1-2 \delta}}{c x / \log ^{2} x} \rightarrow 0$ as $x \rightarrow \infty$ there are a negligible number of primes $p$ such that $\operatorname{ord}(q)=2 p_{1}(\bmod p)$. This fact is also true for $r$ and $s$.

Now, assume that $q$ and $r$ and $s$ have order $2 p_{2}$. Since $\mathbb{F}_{p}^{\star}$ cyclic $\operatorname{group}, \operatorname{ord}(<q, r, s>)=2 p_{2}<x^{3 / 4-\epsilon}$. By lemma 2 in 4 ] the number of primes $p$ such that $\operatorname{ord}(<q, r, s\rangle)<y$ is $O\left(y^{4 / 3}\right)$. So the number of primes $p$ such that $\operatorname{ord}(<q, r, s\rangle)=2 p_{2}<x^{3 / 4-\epsilon}$ is $O\left(x^{1-4 \epsilon / 3}\right)$ and as before, is negligible in comparison to $\frac{c x}{\log ^{2} x}$.

\section{Notation And Preliminaries}

Now before we prove the theorem about the prime divisors of $p+1$ (as in lemma 2.1 for $p-1$ ) we need to decide on some notation.

Let $\Pi(y ; m, s)$ denote the number of primes $p \leq x$ such that $p \equiv$ $s(\bmod m)$ where $m$ and $s$ are some integers, and

$$
E(y ; m, s):=\Pi(y ; m, s)-\frac{\operatorname{Li}(y)}{\varphi(m)}
$$

where $\operatorname{Li}(y)=\int_{2}^{y} \frac{d t}{\log t}$. Also set

$$
E(x ; m):=\max _{1 \leq y \leq x} \max _{(s, m)=1}|E(y ; m, s)| .
$$

Define $\mathcal{A}=\{p+1 \mid p \leq x, p \equiv u(\bmod v)\}$ where $u, v$ are some integers such that $(u, v)=1, u \equiv 1(\bmod 2), 8 \mid v,\left(\frac{u+1}{2}, v\right)=1$ and take $X=\frac{L i(x)}{\varphi(v)}$.

For a square-free integer $d,(d, v)=1$, let

$$
\begin{aligned}
\mathcal{A}_{d} & :=\{a \in \mathcal{A}: a \equiv 0 \bmod d)\} \\
& =\{p+1: p \leq x, p \equiv u \bmod v, p \equiv-1 \quad \bmod d\}
\end{aligned}
$$

By the Chinese remainder theorem there exists an $l$ such that

$$
\left|\mathcal{A}_{d}\right|=\#\{p+1 \mid p \leq x, p \equiv l(\bmod d v)\}
$$

By the definition of $E(x ; d v, l)$,

$$
\begin{gathered}
\left|\mathcal{A}_{d}\right|=\frac{L i x}{\varphi(d v)}+E(x ; d v, l)=\frac{1}{\varphi(d)} \frac{L i x}{\varphi(v)}+E(x ; d v, l)=\frac{X}{\varphi(d)}+E(x ; d v, l) \\
\text { Define } \omega(d):=\frac{d}{\varphi(d)} \text { and } \\
R_{d}:=\left|\mathcal{A}_{d}\right|-\frac{\omega(d)}{d} X=E(x ; d v, l)
\end{gathered}
$$

Finally, we define two arithmetical functions for a square-free $d=p_{1}$. $\cdots p_{k} \cdot \mu(d)=(-1)^{k}$ and $\nu(d)=k($ where $\mu(1)=1$ and $\nu(1)=0)$.

Now we want to prove two lemmas. 
Lemma 3.1. for any prime $q$, which is relatively prime to $v$ we have:

$$
0 \leq \frac{1}{q-1} \leq 1-\frac{1}{c_{1}}
$$

where $c_{1}>1$ is some suitable constant.

$$
\sum_{w \leq q<z} \frac{\log q}{q-1}-\log \frac{z}{w}=O(1) \quad(2 \leq w \leq z)
$$

where $O$ does not depend on $z$ or $w$.

$$
\prod_{\substack{2<q<z \\ q \nmid v}}\left(1-\frac{1}{q-1}\right) \gg \frac{1}{\log z} .
$$

Proof. Since $q>2$ it is clear that (3.1) holds.

As for the second equation, $\sum_{w \leq q<z} \frac{\log q}{q-1}=\sum_{w \leq q<z} \frac{\log q}{q} \frac{q}{q-1}=\sum_{w \leq q<z} \frac{\log q}{q}(1+$ $\left.\frac{1}{q-1}\right)=\sum_{w \leq q<z} \frac{\log q}{q}+\sum_{w \leq q<z} \frac{\log q}{q(q-1)}=\log \frac{z}{w}+O(1) \quad\left(\sum_{p<x} \frac{\log p}{p}=\log x+\right.$ $O(1))$.

Hence we get (3.2). Finally,

$$
\begin{aligned}
\prod_{\substack{2<q<z \\
q \nmid v}}\left(1-\frac{\omega(q)}{q}\right) & =\prod_{\substack{2<q<z \\
q \nmid v}}\left(1-\frac{1}{q-1}\right) \gg \prod_{2<q<z}\left(1-\frac{1}{q-1}\right) \\
& =\exp \left(\log \prod_{2<q<z}\left(1-\frac{1}{q-1}\right)\right) \\
& =\exp \left(\sum_{2<q<z} \log \left(1-\frac{1}{q-1}\right)\right) \\
& \gg \exp \left(\sum_{2<q<z}\left(-\frac{1}{q-1}-\frac{1}{(q-1)^{2}}\right)\right)
\end{aligned}
$$

Since

$$
\frac{1}{q-1}=\frac{1}{q}+\frac{1}{q(q-1)} \leq \frac{1}{q}+\frac{1}{(q-1)^{2}}
$$

and $\sum_{2<q<z} \frac{1}{(q-1)^{2}}$ converges, we get

$$
\prod_{2<q<z}\left(1-\frac{1}{q-1}\right) \gg \exp \left(-\sum_{2<q<z} \frac{1}{q}\right)
$$


Since

we have

$$
\sum_{2<q<z} \frac{1}{q} \sim \log \log z
$$

$$
\exp \left(-\sum_{2<q<z} \frac{1}{q}\right) \gg \exp (-\log \log z)=\frac{1}{\log z}
$$

Lemma 3.2. For any natural square-free number $d,(d, v)=1$, given an $A>0$ there exist constants $c_{2}(\geq 1)$ and $c_{3}(\geq 1)$ such that

$$
\sum_{d<\frac{X^{\frac{1}{2}}}{(\log x)^{c_{2}}}} \mu^{2}(d) 3^{\nu(d)}\left|R_{d}\right| \leq c_{3} \frac{X}{\log ^{A} X}, \quad(X \geq 2)
$$

Proof. denote by $S_{R_{d}}$ the term which we need to estimate.

$$
S_{R_{d}}=\sum_{d<\frac{x^{\frac{1}{2}}}{(\log x)^{c 2}}} \mu^{2}(d) 3^{\nu(d)}\left|R_{d}\right|
$$

By the definitions of $R_{d}$ and $E(x ; d v)$

$$
S_{R_{d}} \leq \sum_{d<\frac{X^{\frac{1}{2}}}{(\log x)^{c 2}}} \mu^{2}(d) 3^{\nu(d)}|E(x ; d v)|
$$

since $E(x ; d v) \ll \frac{x}{d v}$ if $d \leq \frac{x}{v}$ we get that

$$
S_{R_{d}} \ll x^{\frac{1}{2}} \sum_{d<\frac{x^{\frac{1}{2}}}{(\log x)^{c_{2}}}} \frac{\mu^{2}(d) 3^{\nu(d)}}{d^{\frac{1}{2}}}|E(x ; d v)|^{\frac{1}{2}} .
$$

By Cauchy's inequality,

$$
S_{R_{d}} \ll x^{\frac{1}{2}}\left(\sum_{d<X^{\frac{1}{2}}} \frac{\mu^{2}(d) 3^{2 \nu(d)}}{d}\right)^{\frac{1}{2}}\left(\sum_{d v<\frac{v X^{\frac{1}{2}}}{(\log x)^{c_{2}}}}|E(x ; d v)|\right)^{\frac{1}{2}} .
$$

For sufficiently large $x$ we obtain

$$
S_{R_{d}} \ll x^{\frac{1}{2}}\left(\sum_{d<x^{\frac{1}{2}}} \frac{\mu^{2}(d) 3^{2 \nu(d)}}{d}\right)^{\frac{1}{2}}\left(\sum_{d v<\frac{x^{\frac{1}{2}}}{(\log x)^{c 2}}}|E(x ; d v)|\right)^{\frac{1}{2}} .
$$

With Bombieri-Vinogradov theorem ([2]) (given any positive constant $e_{1}$, there exist a positive constant $e_{2}$ such that $\sum E(x ; d)=$

$$
d<\frac{x^{\frac{1}{2}}}{\log ^{e} 2 x}
$$


$\left.O\left(\frac{x}{\log ^{e}{ }^{1} x}\right)\right)$ for the last sum and since $\sum_{d<w} \frac{\mu^{2}(d) 9^{\nu(d)}}{d} \leq(\log w+1)^{9}$ (see 6], p.115, equation (6.7)) we find that for given constant $B$ there exist $c_{2}$ such that

$$
S_{R_{d}} \ll \frac{x}{\log ^{B} x}
$$

So, for given $A$ there exist $c_{2}$ such that

$$
S_{R_{d}} \ll \frac{X}{\log ^{A} X}
$$

where $\ll$ depends on $v$ and $c_{2}$.

\section{Proof of the theorem 1.4 - The Sieve Part}

In this section we will show that for a sufficiently small $0<\delta<1 / 4$ there exists some constant $c(\delta)>0$ (which depends on $\delta$ ) such that for at least $c(\delta) \frac{x}{\log ^{2} x}$ primes $p \leq x, p \equiv u(\bmod v), \frac{p+1}{2}=P_{3}$ where $q \mid \frac{p+1}{2} \Rightarrow q>x^{1 / 4-\delta}$. Later we will sharpen this result further.

4.1. Use of the lower bound linear sieve. In the following subsection we will show, using the linear sieve, that for a sufficiently small $0<\delta<1 / 4$ there exists some constant $c_{1}(\delta)>0$ (which depends on $\delta)$ such that for at least $c_{1}(\delta) \frac{x}{\log ^{2} x}$ primes $p \leq x, p \equiv u(\bmod v), \frac{p+1}{2}$ has at most four prime divisors all of them greater than $x^{\frac{1}{4}-\delta}$.

Define $S(\mathcal{A}, z)=\#\left\{a \mid a \in \mathcal{A},\left(a, \prod_{\substack{p<z \\ p \nmid v}} p\right)=1\right\}$ and let $f$ denote the "lower bound function" for the linear sieve which is defined as $f(t)=$ $2 e^{\gamma} t^{-1} \log (t-1)$ for $2 \leq t \leq 4$, where $\gamma$ is Euler constant. Then (see [6. Theorem 8.4, page 236]):

Lemma 4.1. Assume (3.1), (3.2) and (3.4). Then for $X^{1 / 8}<z<$ $X^{1 / 4}$ we have

$$
S(\mathcal{A}, z) \geq X \prod_{\substack{q<z \\ q \nmid v}}\left(1-\frac{\omega(q)}{q}\right)\left\{f\left(\frac{\log x}{2 \log z}\right)+O\left(\frac{1}{\log x}\right)\right\}
$$

where the O-term does not depend on $X$ or on $z$.

Note 4.2. Obviously $z$ influences the number of primes which divide the elements of $\mathcal{A}$ and their magnitude. Heath-Brown used a stronger version of this lemma which gives $z=x^{1 / 4+\epsilon_{0}}$ where $\epsilon_{0}$ is a specific small real number. 
By Lemmas 3.1] and 3.2, (3.1), (3.2) and (3.4) hold. Hence we can use lemma 4.1] with $z=X^{\frac{1}{4}-\delta}$.

$$
S\left(\mathcal{A}, X^{\frac{1}{4}-\delta}\right) \geq X \prod_{\substack{q<X^{\frac{1}{4}-\delta} \\ q \nmid v}}\left(1-\frac{1}{q-1}\right)\left\{f\left(\frac{1}{2} \frac{\log x}{\log x^{\frac{1}{4}-\delta}}\right)+O\left(\frac{1}{\log x}\right)\right\}
$$

by Lemma 3.1 (3.3) we have

$$
S\left(\mathcal{A}, X^{\frac{1}{4}-\delta}\right) \gg \frac{X}{\log x^{\frac{1}{4}-\delta}} f\left(\frac{2}{1-4 \delta}\right)
$$

But for $2 \leq t \leq 4, f(t)=2 e^{\gamma} t^{-1} \log (t-1)$, and so,

$$
\begin{aligned}
S\left(\mathcal{A}, X^{\frac{1}{4}-\delta}\right) & \gg \frac{X}{\log x^{1 / 4-\delta}} 2 e^{\gamma}\left(\frac{1-4 \delta}{2}\right) \log \frac{1+4 \delta}{1-4 \delta} \\
& \gg \frac{x}{\log ^{2} x} \log \frac{1+4 \delta}{1-4 \delta}=\frac{x}{\log ^{2} x} \log \left(1+\frac{8 \delta}{1-4 \delta}\right) .
\end{aligned}
$$

Since $\log (1+s) / s \sim 1$ as $s \rightarrow 0$ and for, $0<\delta<1 / 4,1-4 \delta$ are bounded, we have:

\section{Lemma 4.3.}

$$
S\left(\mathcal{A}, X^{\frac{1}{4}-\delta}\right) \gg \delta \frac{x}{\log ^{2} x}
$$

where the implied constant in $\gg$ does not depend on $\delta$.

Note 4.4. By definition of $S\left(\mathcal{A}, X^{\frac{1}{4}-\delta}\right)$, for all sufficiently small $0<$ $\delta<1 / 4$, there are at least $\gg \delta \frac{x}{\log ^{2} x}$ primes $p \leq x$, such that any prime divisor of $p+1$ (for a $p$ in our sequence) is greater than $X^{\frac{1}{4}-\delta}$ or divides $v$. Since by our assumption $\left(\frac{u+1}{2}, v\right)=1$ where $p \equiv u(\bmod v)$ and $X=\frac{L i(x)}{\varphi(v)}$ we obtain that all odd prime divisor of $\frac{p+1}{2}$ are greater than $x^{\frac{1}{4}-\delta}$. Hence there are at most four primes divisors of $\frac{p+1}{2}$ which are greater than $x^{\frac{1}{4}-\delta}$. In the next subsection we will show that there is only a small number of primes $p \leq x$ such that $\frac{p+1}{2}$ has exactly four primes divisors all of which are greater than $x^{\frac{1}{4}-\delta}$.

4.2. First use of the Selberg upper bound sieve. In order to prove that there is only a small number of primes $p \leq x$ such that exactly four primes divide $\frac{p+1}{2}$ we need to use Selberg's upper bound sieve (see [6, theorem 3.12]):

Proposition 4.5. Let $a, b$ be integers satisfying

$$
a b \neq 0, \quad \operatorname{gcd}(a, b)=1, \quad 2 \mid a b
$$


Then as $x \rightarrow \infty$ we have uniformly in $a, b$ that

$$
\begin{aligned}
& \mid\{p: p \leq x, \text { ap }+b=\text { prime }\} \mid \leq \\
& 8 \prod_{p>2}\left(1-\frac{1}{(p-1)^{2}}\right) \prod_{2<p \mid a b} \frac{p-1}{p-2} \frac{x}{\log ^{2} x}\left\{1+O\left(\frac{\log \log x}{\log x}\right)\right\}
\end{aligned}
$$

From this proposition we derive the following:

Lemma 4.6. For any $0<\delta<1 / 4$, there exists $c_{2}(\delta) \frac{x}{\log ^{2} x}\left(c_{2}(\delta)>0\right)$ primes $p \leq x$ such that $\frac{p+1}{2}$ has at most three prime divisors all of which are greater than $x^{1 / 4-\delta}$.

Proof. Assume that $\frac{p+1}{2}=p_{1} p_{2} p_{3} p_{4}, p \leq x$ where the $p_{i} s$ primes greater than $x^{1 / 4-\delta}$. Instead of counting the elements in this set we can count the products of primes $p_{1} p_{2} p_{3} p_{4}$ such that $2 p_{1} p_{2} p_{3} p_{4}-1=p \leq x$ where the $p_{i} s$ are primes greater than $x^{1 / 4-\delta}$.

To count the latter set we use proposition 4.5. We take $a=2 p_{1} p_{2} p_{3}$, $b=-1$ and $Y=\frac{x+1}{2 p_{1} p_{2} p_{3}}\left(\right.$ since $\left.2 p_{1} p_{2} p_{3} p_{4}-1 \leq x \Leftrightarrow p_{4} \leq \frac{x+1}{2 p_{1} p_{2} p_{3}}\right)$.

By the proposition,

$$
\begin{aligned}
S_{p_{4}}=\#\left\{p_{4} \leq Y: a p_{4}+b=\text { prime }\right\} & \\
=\#\left\{p_{4} \leq \frac{x+1}{2 p_{1} p_{2} p_{3}}:\right. & \left.2 p_{1} p_{2} p_{3} p_{4}-1=\text { prime }\right\} \\
& \ll \frac{x+1}{2 p_{1} p_{2} p_{3} \log ^{2} \frac{x+1}{2 p_{1} p_{2} p_{3}}} \prod_{\substack{p \mid 2 p_{1} p_{2} p_{3} \\
p \neq 2}} \frac{p-1}{p-2}
\end{aligned}
$$

Since the $p_{i}$ 's are big primes, the term $\prod_{\substack{p \mid 2 p_{1} p_{2} p_{3} \\ p \neq 2}} \frac{p-1}{p-2}$ is approximately one. Then

$$
S_{p_{4}} \ll \frac{x+1}{2 p_{1} p_{2} p_{3} \log ^{2} \frac{x+1}{2 p_{1} p_{2} p_{3}}}
$$

From the fact that for all $i=1,2,3, p_{i}<x^{1 / 4+3 \delta}$ we have for a sufficiently small $\delta$

$$
\begin{aligned}
S_{p_{4}} & \ll \frac{1}{p_{1} p_{2} p_{3} \log ^{2} \frac{x}{\left(x^{1 / 4+3 \delta}\right)^{3}}} \\
& \ll \frac{1}{p_{1} p_{2} p_{3}} \cdot \frac{x}{\log ^{2} x^{1 / 4-9 \delta}} \ll\left(\frac{1}{1 / 4-9 \delta}\right)^{2} \frac{1}{p_{1} p_{2} p_{3}} \cdot \frac{x}{\log ^{2} x}
\end{aligned}
$$


Now we shall sum-up the last term over all possibilities for $p_{1}, p_{2}, p_{3}$. This number is bounded by

$$
S_{p_{4}}^{*}=4 \frac{x}{\log ^{2} x} \sum_{p_{1}} \frac{1}{p_{1}} \sum_{p_{2}} \frac{1}{p_{2}} \sum_{p_{3}} \frac{1}{p_{3}}
$$

where the sum is over $x^{1 / 4-\delta}<p_{i}<x^{1 / 4+3 \delta}, i=1,2,3$.

Observation 4.7. We have $\sum_{x^{\beta}<p<x^{\alpha}} \frac{1}{p}=\log \frac{\alpha}{\beta}+o(1)$.

By observation 4.7

$$
S_{p_{4}}^{*} \ll \log ^{3} \frac{1 / 4+3 \delta}{1 / 4-\delta} \frac{x}{\log ^{2} x} \ll \log ^{3}\left(1+\frac{16 \delta}{1-4 \delta}\right) \frac{x}{\log ^{2} x}
$$

Since $\log (1+s)=O(s)$ for $0<s<1$ and for, $0<\delta<1 / 4,1-4 \delta$ is bounded, we have

$$
S_{p_{4}}^{*} \ll \delta^{3} \frac{x}{\log ^{2} x}
$$

where $\ll$ does not depend on $\delta$. Hence, $S_{p_{4}}^{*}$ is a small number in comparison to $S\left(\mathcal{A}, X^{\frac{1}{4}-\delta}\right) \gg \delta \frac{x}{\log ^{2} x}$.

4.3. Second use of Selberg's upper bound sieve. Up till now we know that for any sufficiently small number $\delta>0$, there are $c_{2}(\delta) \frac{x}{\log ^{2} x}$ primes $p \leq x$ such that $\frac{p+1}{2}$ has at most three prime divisors all of which are greater than $x^{1 / 4-\delta}$. In this section we want to prove the existence of $c_{3}(\delta) \frac{x}{\log ^{2} x}$ primes $p \leq x$, such that $\frac{p+1}{2}=P_{3}$ and if $\frac{p+1}{2}$ is a product of exactly three primes $q_{3} \geq q_{2} \geq q_{1}$ then $q_{1}>x^{1 / 4-\delta}, q_{2}>x^{1 / 4+2 \delta}, q_{3}>$ $x^{1 / 3+\delta^{2}}$. First we prove the claim about $q_{2}$ (by the previous subsections it is clear that $\left.q_{1}>x^{1 / 4-\delta}\right)$.

Assume that $q_{1}$ and $q_{2}$ take values between $x^{\frac{1}{4}-\delta}$ and $x^{\frac{1}{4}+2 \delta}$. Instead of count the number of primes $p \leq x$ such that $\frac{p+1}{2}=q_{1} q_{2} q_{3}$ where $q_{1}$ and $q_{2}$ are between $x^{\frac{1}{4}-\delta}$ and $x^{\frac{1}{4}+2 \delta}$ we shall count the products $q_{1} q_{2} q_{3}$ such that $2 q_{1} q_{2} q_{3}-1=p \leq x$ where $q_{1}$ and $q_{2}$ are between $x^{\frac{1}{4}-\delta}$ and $x^{\frac{1}{4}+2 \delta}$.

To count this set we use Proposition 4.5. Define $a=2 q_{1} q_{2}, b=-1$ and $Y=\frac{x+1}{2 q_{1} q_{2}}$ (since $2 q_{1} q_{2} q_{3}-1 \leq x \Leftrightarrow q_{3} \leq \frac{x+1}{2 q_{1} q_{2}}$ ). By Proposition 4.5

$$
\begin{aligned}
S_{q_{3}} & =\#\left\{q_{3} \leq Y: a q_{3}+b=\text { prime }\right\} \\
& =\#\left\{q_{3} \leq \frac{x+1}{2 q_{1} q_{2}}: 2 q_{1} q_{2} q_{3}-1=\text { prime }\right\} \\
& \ll \frac{x+1}{2 q_{1} q_{2} \log ^{2} \frac{x+1}{2 q_{1} q_{2}}} \prod_{\substack{p \mid 2 q_{1} q_{2} \\
p \neq 2}} \frac{p-1}{p-2}
\end{aligned}
$$


As in the previous subsection, since the $q_{i}{ }^{6} s$ are big primes the term $\prod_{\substack{p \mid 2 q_{1} q_{2} \\ p \neq 2}} \frac{p-1}{p-2}$ is approximately one, so

$$
S_{q_{3}} \ll \frac{x+1}{2 q_{1} q_{2} \log ^{2} \frac{x+1}{2 q_{1} q_{2}}} .
$$

Now we sum-up the last term over all possibilities for $q_{1}, q_{2}$. This number bound by, (see the last previous subsection).

$$
S_{q_{3}}^{*}=\frac{x}{\log ^{2} x} \sum_{x^{\frac{1}{4}-\delta} \leq q_{1} \leq x^{\frac{1}{4}+2 \delta}} \frac{1}{q_{1}} \sum_{x^{\frac{1}{4}-\delta} \leq q_{2} \leq x^{\frac{1}{4}+2 \delta}} \frac{1}{q_{2}} \ll \frac{x}{\log ^{2} x} \log ^{2} \frac{1+8 \delta}{1-4 \delta}
$$

Since $\log ^{2} \frac{1+8 \delta}{1-4 \delta}=O\left(\delta^{2}\right), S_{q_{3}}^{*}=O\left(\delta^{2} \frac{x}{\log ^{2} x}\right)$. Hence for any $\delta$ sufficiently small we get a small number of primes $p \leq x$ such that $\frac{p+1}{2}=q_{1} q_{2} q_{3}$ where $q_{1}$ and $q_{2}$ are between $x^{\frac{1}{4}-\delta}$ and $x^{\frac{1}{4}+2 \delta}$. Thus for most such $p$, we have $q_{2}>x^{1 / 4+2 \delta}$.

Finally we prove the claim about $q_{3}$. Assume that $\frac{p+1}{2}=q_{1} q_{2} q_{3}, q_{3} \geq$ $q_{2} \geq q_{1}$ then we have that $q_{3} \geq\left(\frac{p+1}{2}\right)^{\frac{1}{3}}$. The following lemma sharpens this result.

Lemma 4.8. For any $0<\delta<1 / 4$ there are at most $O\left(\delta^{2} \frac{x}{\log ^{2} x}\right)$ primes $p \leq x$ for which $\left(\frac{p+1}{2}\right)^{\frac{1}{3}} \leq q_{3} \leq p^{\frac{1}{3}+\delta^{2}}$ where $O$ does not depend on $\delta$.

Proof. Note that if $\frac{p+1}{2} \geq \frac{x}{\log ^{2} x}$ then $q_{3} \geq\left(\frac{p+1}{2}\right)^{\frac{1}{3}} \geq\left(\frac{x}{\log ^{2} x}\right)^{\frac{1}{3}} \geq x^{\frac{1}{3}-\delta^{2}}$ for $x \geq x(\delta)$ (the number of primes $p$ for which $\frac{p+1}{2} \leq \frac{x}{\log ^{2} x}$, is $o\left(\frac{x}{\log ^{2} x}\right)$ by prime number theorem and so may be ignored).

Assume now that $\frac{p+1}{2}=q_{1} q_{2} q_{3}$ with $x^{1 / 3-\delta^{2}} \leq q_{3} \leq x^{1 / 3+\delta^{2}}$ and $x^{1 / 4+2 \delta} \leq q_{2} \leq x^{5 / 12+\delta+\delta^{2}}$ (this is the maximum range which $q_{2}$ can be in). Using proposition 4.5, we take $a=2 q_{2} q_{3}, b=-1, Y=\frac{x+1}{2 q_{2} q_{3}}$, and so

$$
\begin{aligned}
S_{q_{1}} & =\#\left\{q_{1} \leq Y: a q_{1}+b=\text { prime }\right\} \\
& =\#\left\{q_{1} \leq \frac{x+1}{2 q_{2} q_{3}}: 2 q_{2} q_{3}-1=\text { prime }\right\} \\
& \ll \frac{x+1}{2 q_{2} q_{3} \log ^{2} \frac{x+1}{2 q_{2} q_{3}}} \prod_{\substack{p \mid 2 q_{2} q_{3} \\
p \neq 2}} \frac{p-1}{p-2}
\end{aligned}
$$

Since $2 x^{3 / 4+\delta+2 \delta^{2}}$ is the maximum of $2 q_{2} q_{3}\left(q_{1}>x^{1 / 4-\delta}\right)$ we obtain

$$
S_{q_{1}} \ll \frac{x}{2 q_{2} q_{3} \log ^{2} \frac{x}{x^{3 / 4+\delta}}} \ll \frac{x}{q_{2} q_{3} \log ^{2} x}
$$


Now we sum-up the last term over all possibilities for $q_{2}, q_{3}$. this number is bounded by, (see in the proof of lemma 4.6)

$$
\begin{aligned}
S_{q_{1}}^{*} & =\frac{x}{\log ^{2} x} \sum_{x^{\frac{1}{4}+2 \delta} \leq q_{2} \leq x^{\frac{5}{12}}+\delta+\delta^{2}} \frac{1}{q_{2}} \sum_{x^{\frac{1}{3}-\delta^{2} \leq q_{3} \leq x^{\frac{1}{3}+\delta^{2}}}} \frac{1}{q_{3}} \\
& \ll \frac{x}{\log ^{2} x} \log \frac{5 / 12+\delta+\delta^{2}}{1 / 4+2 \delta} \log \frac{1 / 3+\delta^{2}}{1 / 3-\delta^{2}} \\
& \ll \frac{x}{\log ^{2} x} \log \left(1+\frac{6 \delta^{2}}{1-3 \delta^{2}}\right)=O\left(\delta^{2} \frac{x}{\log ^{2} x}\right)
\end{aligned}
$$

and for a sufficiently small $\delta$ we can ignore this number.

By the same method (see lemma 3 in [8] there are only $O\left(\delta^{2} \frac{x}{\log ^{2} x}\right)$ primes $p \leq x$ such that $\frac{p+1}{2}=r_{1} r_{2}$ where $r_{i}{ }^{6} s$ are primes, $i=1,2, r_{2} \geq$ $r_{1}, \quad p^{1 / 2-\delta^{2}} \leq r_{1} \leq\left(\frac{p+1}{2}\right)^{1 / 2}$.

If we summarize this section we conclude that for any sufficiently small $0<\delta<1 / 4$ there are at least $c_{3}(\delta) \frac{x}{\log ^{2} x}, \quad c_{3}(\delta)>0$, primes $p \leq x, \quad p \equiv u(\bmod v)$ such that we can factor $\frac{p+1}{2}$ in at least one of the following options:

(1) $\frac{p+1}{2}$ is a prime number.

(2) $\frac{p+1}{2}=r_{1} r_{2}$ where $r_{1}, r_{2}$ are some prime numbers, $p^{1 / 4-\delta}<r_{1}<$ $p^{1 / 2-\delta^{2}}, \quad p^{1 / 2+\delta^{2}}<r_{2}<p^{3 / 4+\delta}$.

(3) $\frac{p+1}{2}=q_{1} q_{2} q_{3}$ where $q_{1} \leq q_{2} \leq q_{3}$ are some prime numbers, $q_{1}>p^{1 / 4-\delta}, \quad q_{2}>p^{1 / 4+2 \delta}, \quad q_{3}>p^{1 / 3+\delta^{2}}$ and so $p^{1 / 2+\delta}<$ $q_{1} q_{2}<p^{2 / 3-\delta^{2}}, \quad p^{7 / 12-\delta+\delta^{2}}<q_{1} q_{3}<p^{3 / 4-2 \delta}, \quad p^{7 / 12+2 \delta+\delta^{2}}<$ $q_{2} q_{3}<p^{3 / 4+\delta}$.

\section{Proof of the theorem - The Algebraic Part}

5.1. Construction of the arithmetic sequence. In this section we want to construct integers $u$ and $v,(u, v)=1$ such that for all primes $p$ such that $p \equiv u(\bmod v)$, the discriminants $\Delta_{1}, \ldots, \Delta_{7}, \Delta_{i} \neq 3$ of $\mathbb{Q}\left(\sqrt{\Delta_{1}}\right), \ldots, \mathbb{Q}\left(\sqrt{\Delta_{7}}\right)$, respectively, satisfy

$$
\left(\frac{\Delta_{1}}{p}\right)=\left(\frac{\Delta_{2}}{p}\right)=\ldots=\left(\frac{\Delta_{7}}{p}\right)=-1 .
$$

This means that $p$ is inert simultaneously in all of the fields.

In addition we want to insure that $\frac{p+1}{2}$ will be an odd integer and so we take $u \equiv 1(\bmod 4)$ where $8 \mid v$. Finally, to get $\left(\frac{p+1}{2}, v\right)=1$ we shall construct $u$ and $v$ so that $\left(\frac{u+1}{2}, v\right)=1$ (since after sieving the small factors of $\frac{p+1}{2}$ we may be left with small factors which divide $v$, see previous section). 
In order to fulfill these demands, we will first show that there exist infinitely many primes $p$ with the following simultaneous conditions

$$
\left(\frac{-1}{p}\right)=\left(\frac{3}{p}\right)=1 \text { and }\left(\frac{\Delta_{1}}{p}\right)=\left(\frac{\Delta_{2}}{p}\right)=\ldots=\left(\frac{\Delta_{7}}{p}\right)=-1
$$

This condition is equivalent to the condition:

$$
B(p)=\left(1+\left(\frac{-1}{p}\right)\right)\left(1+\left(\frac{3}{p}\right)\right)\left(1-\left(\frac{\Delta_{1}}{p}\right)\right) \cdots\left(1-\left(\frac{\Delta_{7}}{p}\right)\right) \neq 0
$$

Since the Legendre symbol is a multiplicative function, we obtain,

$$
B(p)=\left(1+\left(\frac{-1}{p}\right)\right)\left(1+\left(\frac{3}{p}\right)\right)\left(1-\Sigma\left(\frac{\Delta_{i}}{p}\right)+\Sigma\left(\frac{\Delta_{i} \Delta_{j}}{p}\right)-\ldots-\left(\frac{\Delta_{1} \cdots \Delta_{7}}{p}\right)\right)
$$

Let $S$ be the set of all integers of the form $n=(-1)^{a_{1}} 3^{a_{2}} \prod_{i=1}^{7} \Delta_{i}^{b_{i}}, a_{i}, b_{i} \in$ $\{0,1\}$. Then

$$
\sum_{p \leq Z} B(p)=\sum_{n \in S}(-1)^{b_{1}+\ldots+b_{7}} \sum_{p \leq Z}\left(\frac{n}{p}\right) \quad b_{i} \in\{0,1\}
$$

By the assumption in the theorem (see the introduction) each $n \in S$ is not a square when $\sum_{i=1}^{7} b_{i}$ is odd.

This assumption with the fact that for $n$ not a perfect square (by reciprocity law for Legendre symbol)

$$
\sum_{p \leq Z}\left(\frac{n}{p}\right)=o(\pi(Z)) \text { as } Z \rightarrow \infty
$$

implies that $\sum_{p \leq Z} B(p)$ is asymptotic to at least $\pi(Z)$ (since all the negative summands contribute $o(\pi(Z))$ and at least the natural number 1 contributes $\pi(Z))$. This shows that the simultaneous conditions have infinitely many solutions $p$.

We fix some particular $p_{0}$ satisfying the condition. We define $u_{2}=p_{0}$ and for each odd prime $l$, such that $l \mid \Delta_{1} \cdots \Delta_{7}$ we define $u_{l}=p_{0}$ if $l \nmid p_{0}+1$ and $u_{l}=4 p_{0}$ otherwise.

Claim 5.1. $l \nmid u_{l}+1$

Proof. If $u_{l}=p_{0}$ then by the assumption $l \nmid p_{0}+1$, so $l \nmid u_{l}+1$. If $u_{l}=4 p_{0}$, assume, by reductio ad absurdum, that $l \mid u_{l}+1$. Hence $l \mid 4 p_{0}+1$. Because, $u_{l}=4 p_{0}$ and $l \mid p_{0}+1$, we obtain that $l \mid 3 p_{0}$. On the other hand, by our condition, $\left(\frac{3}{p_{0}}\right)=1$ so $\left(\frac{p_{0}}{3}\right)=1\left(p_{0} \equiv 1(\bmod 4)\right)$. Hence $p_{0} \equiv 1(\bmod 3)$. Since $l \mid p_{0}+1$ and $p_{0} \equiv 1(\bmod 3)$ we conclude that $l \nmid 3$. Using the assumption that $l \mid p_{0}+1$ we deduce that $l \neq p_{0}$ (if $l=p_{0}$ then $l \nmid p_{0}+1$ ). Hence $l \nmid 3 p_{0}$, a contradiction. 
Let $v=8 \Delta_{1} \cdots \Delta_{7}$ and $u$ be the common solution of $u \equiv u_{2}(\bmod 8)$ and all the congruences $u \equiv u_{l}(\bmod l)$. Such a solution exists, by the Chinese Remainder Theorem.

Since $l \nmid u+1$ for every odd prime $l \mid v$ and the fact that $u \equiv 1(\bmod 4)$. (by the construction $u \equiv u_{2}(\bmod 8)$ where $\left.u_{2}=p_{0} \equiv 1(\bmod 4)\right)$ we conclude that $\left(\frac{u+1}{2}, v\right)=1$. Finally, if $p \equiv u(\bmod v)$ then, $p \equiv$ $p_{0}(\bmod 8)$ and $p \equiv p_{0}$ or $4 p_{0}(\bmod l)$ for all odd primes $l \mid v$. So, $\left(\frac{\Delta_{1}}{p}\right)=\left(\frac{\Delta_{1}}{p_{0}}\right)=-1$, and similarly for all $\Delta_{i}$ 's. This completes the construction of $u$ and $v$.

Note that by the construction of the integers $u$ and $v$ we have that $(u, v)=1$. (take $l$ an odd prime number, $l \mid v=8 \Delta_{1} \cdots \Delta_{7}$ and assume that $l \mid u$. Since $u \equiv u_{l}(\bmod l)$ then $l \mid u_{l}$ hence $l \mid p_{0}$ or $4 p_{0}$ in other words $l=p_{0}$. But $p_{0} \nmid \Delta_{1} \cdots \Delta_{7}$ ( $p_{0}$ fulfills the simultaneous condition (5.1) ) and $\left.l \mid \Delta_{1} \cdots \Delta_{7}\right)$.

5.2. The last step of the proof. For the last step of the proof we need to use lemma 4 from Narkiewicz [13, which generalized lemma 2 in [4].

Lemma 5.2. If $a_{1}, \ldots a_{k}$ are multiplicatively independent integers of an algebraic number-field $K, G$ the subgroup of $K^{\star}$ generated by $a_{1}, \ldots a_{k}$, and for any prime ideal $\mathbf{P}$ not dividing $a_{1}, \cdots a_{k}$ we denote by $G_{\mathbf{P}}$ the reduction of $G(\bmod \mathbf{P})$, then for all positive $y$ one can have $\# G_{\mathbf{P}}<y$ for at most $O\left(y^{1+\frac{1}{k}}\right)$ prime ideals $\mathbf{P}$, with the implied constant being dependent on the $a_{i}$ 's and $K$.

Now, as we saw at the end of section 3, for any sufficiently small $0<\delta<1 / 4$ there is some constant $c_{3}(\delta)>0$ such that for $c_{3}(\delta) \frac{x}{\log ^{2} x}$ primes $p \leq x, p \equiv u(\bmod v)$ at least one of the following occur:

(1) $\frac{p+1}{2}$ is a prime number.

(2) $\frac{p+1}{2}=r_{1} r_{2}$ where $r_{1}, r_{2}$ are primes so that, $p^{1 / 4-\delta}<r_{1}<$ $p^{1 / 2-\delta^{2}}, \quad p^{1 / 2+\delta^{2}}<r_{2}<p^{3 / 4+\delta}$.

(3) $\frac{p+1}{2}=q_{1} q_{2} q_{3}$ where $q_{1} \leq q_{2} \leq q_{3}$ are primes such that, $p^{1 / 2+\delta}<$ $q_{1} q_{2}<p^{2 / 3-\delta^{2}}, p^{7 / 12-\delta+\delta^{2}}<q_{1} q_{3}<p^{3 / 4-2 \delta}, p^{7 / 12+2 \delta+\delta^{2}}<q_{2} q_{3}<$ $p^{3 / 4+\delta}$.

It is clear by the construction of $u$ and $v$ that $p \equiv 1(\bmod 4)$. Because $\# C_{\epsilon}(p)=p+1$ when $p$ is inert in $\mathbb{Q}(\sqrt{\Delta})$ the unit -1 is a non-square in the group $C_{\epsilon}(p)$. Hence for any unit $\epsilon$, we can choose constant $c= \pm 1$ such that $c \epsilon$ is a non-square in $C_{\epsilon}(p)$. Similarly, since $c \epsilon$ is a non-square and the index of the group of squares is 2 , by the theorem on cyclic groups, the order of $c \epsilon$ is even. 
Now we look at our cases:

(1) In this case, by the above note, $c \epsilon$, if not primitive, has order 2 But the number of $p^{6} \mathrm{~s}$ with this property is $\mathrm{O}(1)$ (by lemma 5.2).

(2) Let $c_{1} \epsilon_{1}, \ldots, c_{4} \epsilon_{4}$ be units in the orders $\mathcal{O}_{\Delta_{1}}, \ldots, \mathcal{O}_{\Delta_{4}}$ of $\mathbb{Q}\left(\sqrt{\Delta_{1}}\right)$, $\ldots, \mathbb{Q}\left(\sqrt{\Delta_{4}}\right), \Delta_{i} \neq 3, i=1,2,3,4$, respectively. We will show that one of them is primitive infinitely many times.

If $\operatorname{ord}\left(c_{i} \epsilon_{i}\right)=2 r_{1}<2 x^{1 / 2-\delta^{2}}$ for some $i=1,2,3,4$. by Lemma 5.2 this occurs in at most $O\left(x^{1 / 2-\delta^{2}}\right)^{2}=x^{1-2 \delta^{2}}$ and this is a negligible number compared to $c_{3}(\delta) \frac{x}{\log ^{2} x}$.

Assume ord $\left(c_{i} \epsilon_{i}\right)=2 r_{2}, i=1,2,3,4$. Consider the ring of integers $\mathcal{O}_{M}$ of the compositum field $M$ of $\mathbb{Q}\left(\sqrt{\Delta_{i}}\right) i=1, \ldots, 4$.

Proposition 5.3. For any prime ideal $P \mid(p)=p \mathcal{O}_{M}$ :

$$
\left(\mathcal{O}_{M} / \mathbf{P}\right)^{\star} \simeq\left(\mathcal{O}_{\Delta_{1}} / p \mathcal{O}_{\Delta_{1}}\right)^{\star} \simeq \ldots \simeq\left(\mathcal{O}_{\Delta_{4}} / p \mathcal{O}_{\Delta_{4}}\right)^{\star}
$$

Proof. Since $p$ is inert, the order of $\mathcal{O}_{\Delta_{i}} / p \mathcal{O}_{\Delta_{i}}$ is $p^{2}$, i.e., $\left[\mathcal{O}_{\Delta_{i}} / p \mathcal{O}_{\Delta_{i}}\right.$ : $\mathbb{Z} / p \mathbb{Z}]=2$. Since all these quotient fields are finite fields and two finite fields with the same number of elements are isomorphic, it is enough to show that,

$$
f=\left[\mathcal{O}_{M} / P: \mathbb{Z} / p \mathbb{Z}\right]=2 .
$$

Consider the Galois group $G=\operatorname{Gal}[M / \mathbb{Q}]$ and define two subgroups of $G$, the decomposition group $D$ and the inertia group $E$ :

$$
D=D(P \mid(p))=\{\sigma \in G \mid \sigma(P)=P\}
$$

and

$$
E=E(P \mid(p))=\{\sigma \in G \mid \sigma(\alpha) \equiv \alpha(\bmod P), \forall \alpha \in \mathbb{Z}\} .
$$

Now, consider the Galois group $\bar{G}$,

$$
\bar{G}=\operatorname{Gal}\left[\mathcal{O}_{M} / P / \mathbb{Z} / p \mathbb{Z}\right]
$$

By [12, chapter 4, beginning],

$$
D / E \simeq \bar{G}
$$

By theorem 28 in in 12 ( since $(p)$ is inert in all the fields $\mathbb{Q}\left(\sqrt{\Delta_{i}}\right),(p)$ is unramified in all the fields $\mathbb{Q}\left(\sqrt{\Delta_{i}}\right)$. Hence $(p)$ is also unramified in $\mathcal{O}_{M}$, i.e., $e$ - the exponent of $P$ in the decomposition of $(p)$, equal to 1$)$

$$
\begin{gathered}
|E|=e=1 \\
|D|=f
\end{gathered}
$$

Immediately we conclude that,

$$
D \simeq \bar{G}
$$


Since $\bar{G}$ is a cyclic group, we get that $D$ is a cyclic group of order $f$. But $D$ is a subgroup of $G$ and $G=C_{2} \times \ldots \times C_{2}$ where $C_{2}$ is a group of order 2. So, $f \leq 2$. Since

$$
\left[\mathcal{O}_{\Delta_{i}} / p \mathcal{O}_{\Delta_{i}}: \mathbb{Z} / p \mathbb{Z}\right]=2
$$

we also see that $f \geq 2$. Hence $f=2$.

Because the compositum of normal extensions is normal, this claim is true for all the prime ideals $P$ in the decomposition of $(p)$ (they have the same $e$ and the same $f$ )

By the last proposition $\left|<c_{1} \epsilon_{1}, c_{2} \epsilon_{2}, c_{3} \epsilon_{3}, c_{4} \epsilon_{4}>\right|=2 r_{2} \ll p^{3 / 4+\delta}$ in $\left(\mathcal{O}_{M} / \mathbf{P}\right)^{\star}$ for $(p) \mid P$ (this is a cyclic group). By Lemma 5.2 the number of $p \leq x$ that have this order is at most $O\left(x^{3 / 4+\delta}\right)^{\frac{5}{4}}=O\left(x^{15 / 16+5 \delta / 4}\right)$ but we can choose $\delta$ to be as small as needed. Hence the set of primes $p, p \leq x$ such that $c_{i} \epsilon_{i} i=1, \ldots, 4$ have order $2 r_{2}$ is a small in comparison with $c_{3}(\delta) \frac{x}{\log ^{2} x}$.

(3) As we did in part (2), we define $c_{i} \epsilon_{i}, c_{i}= \pm 1$ in the ring of integers $\mathcal{O}_{\Delta_{i}}$ of $\mathbb{Q}\left(\sqrt{\Delta_{i}}\right), \Delta_{i} \neq 3, i=1, \ldots, 7$, respectively. Assume that each has order $2 q_{j}<2 x^{1 / 2-\delta}, j=1,2$ or 3 . By lemma 5.2 at most $O\left(x^{1-2 \delta}\right)$ primes $p \leq x$ have this property. Now, let's take any two units of the seven units above, $c_{j} \epsilon_{j}$ and $c_{k} \epsilon_{k} \quad 1 \leq j, k \leq 7$ and assume that they have order $2 q_{1} q_{2}<2 x^{2 / 3-\delta^{2}}$. By lemma 5.2 we can prove (in the same way we did in $(2))$, that this occurs for at most $O\left(x^{1-3 / 2 \delta^{2}}\right)$ primes $p \leq x$.

To summarize, we take $\delta>0$ such that for $c_{3}(\delta) \frac{x}{\log ^{2} x}$ primes $p \leq x$, $c_{1} \epsilon_{1}, \ldots, c_{6} \epsilon_{6}$ do not have order $2 q_{j}, j=1,2,3$ and $2 q_{1} q_{2}$.

From these six units we take three, $c_{i_{1}} \epsilon_{i_{1}}, c_{i_{2}} \epsilon_{i_{2}}, c_{i_{3}} \epsilon_{i_{3}}$ and assume that they have order $2 q_{1} q_{3}<2 x^{3 / 4-2 \delta}$. Again by lemma 5.2 this occurs for at most $O\left(x^{1-8 / 3 \delta}\right)$ primes $p \leq x$. In other words at least four of them do not have order $2 q_{1} q_{3}$ for $c_{3}(\delta) \frac{x}{\log ^{2} x}$ primes.

Consider these four units $c_{1} \epsilon_{1}, \ldots, c_{4} \epsilon_{4}$ (without loss generality) and assume that they have an order $2 q_{2} q_{3}<2 x^{3 / 4+\delta}$. Again by lemma 5.2 this occur in at most $O\left(x^{15 / 16+5 / 4 \delta}\right)$ primes $p \leq x$. So, for a sufficiently small $0<\delta<1 / 4$, there is at least one unit, say $c_{1} \epsilon_{1}$, such that for $c_{3}(\delta) \frac{x}{\log ^{2} x}$ primes $p \leq x, c_{1} \epsilon_{1}$ is primitive.

Note that this theorem implies Corollary 1.5 
Acknowledgement I wish to gratefully acknowledge my Ph.D supervisors Prof. Zee'v Rudnick and Prof. Jack Sonn for the helpful suggestion and the fruitful ideas.

\section{REFERENCES}

[1] E. Artin, Collected Papers, Reading, MA:Addison-Wesley (1965).

[2] E. Bombieri, On the large sieve, Mathematika 12 (1965), 201-225.

[3] G. Cooke and P. J. Wienberger, On the construction of division chains in algebraic number rings, with applications to $S L_{2}$, Comm. Algebra 3 (1975), 481-524.

[4] R. Gupta and R. Murty, A remark on Artin's conjecture, Invent. Math. 78, 127-130 (1984).

[5] R. Gupta, V. Kumar Murty and M. Ram Murty, The Euclidean algorithm for $S$ integers, CNS Conference Proceedings, Vol.7 (1985), 189-202.

[6] Halberstam and Richert, Sieve Methods, Academic Press, London 1974.

[7] D. R. Heath-Brown, Artin's conjecture for primitive roots, Quart. J. Math. Oxford (2), 37 (1986), 27-38.

[8] C. Hooley, On Artin's Conjecture, J. Reine Angew. Math. 226 (1967), 209-220.

[9] P. Kurlberg and Z. Rudnick, Hecke theory and equidistribution for the quantization of linear maps of the torus, Duke Math. Jour. 103 (2000), 47-77.

[10] P. Kurlberg and Z. Rudnick, On quantum ergodicity for linear maps of the torus, Comm. in Math. Physics. 222 (2001) 1, 201-227.

[11] H. W. Lenstra, Jr., On Artin's conjecture and Euclid's algorithm in global fields, Invent. Math. 42, (1977), 201-224.

[12] D. Marcus, Number Fields, Springer, New York 1977.

[13] W. Narkiewicz, A note on Artin's conjecture in algebraic number fields, J. Reine Angew. Math. 381 (1987), 110-115.

[14] W. Narkiewicz, Units in residue classes, Arc. Math., Vol. 51, 238-241 (1988)

[15] H. Roskam, Artin's primitive root conjecture for quadratic fields, J. Number Theory 81(2000), no 1, 93-109.

[16] O. Taussky, Introduction into connections betwween algebraic number theory and integral matrices, Appendix to $\mathrm{H}$. Cohn A Classical Invitation to Algebraic 
Numbers and Class Fields, Springer, New York 1978.

E-mail address: coheny@techunix.technion.ac.il

Math Department, Technion, Haifa, 32000, Israel 